\title{
Cross-validated stepwise regression for identification of novel non-nucleoside reverse transcriptase inhibitor resistance associated mutations
}

Koen Van der Borght ${ }^{1,2^{*}}$, Elke Van Craenenbroeck ${ }^{1}$, Pierre Lecocq ${ }^{1}$, Margriet Van Houtte ${ }^{1}$, Barbara Van Kerckhove ${ }^{1}$, Lee Bacheler ${ }^{3}$, Geert Verbeke ${ }^{2}$ and Herman van Vlijmen ${ }^{1}$

\begin{abstract}
Background: Linear regression models are used to quantitatively predict drug resistance, the phenotype, from the HIV-1 viral genotype. As new antiretroviral drugs become available, new resistance pathways emerge and the number of resistance associated mutations continues to increase. To accurately identify which drug options are left, the main goal of the modeling has been to maximize predictivity and not interpretability. However, we originally selected linear regression as the preferred method for its transparency as opposed to other techniques such as neural networks. Here, we apply a method to lower the complexity of these phenotype prediction models using a 3-fold cross-validated selection of mutations.

Results: Compared to standard stepwise regression we were able to reduce the number of mutations in the reverse transcriptase (RT) inhibitor models as well as the number of interaction terms accounting for synergistic and antagonistic effects. This reduction in complexity was most significant for the non-nucleoside reverse transcriptase inhibitor (NNRTI) models, while maintaining prediction accuracy and retaining virtually all known resistance associated mutations as first order terms in the models. Furthermore, for etravirine (ETR) a better performance was seen on two years of unseen data. By analyzing the phenotype prediction models we identified a list of forty novel NNRTI mutations, putatively associated with resistance. The resistance association of novel variants at known NNRTI resistance positions: 100, 101, 181, 190, 221 and of mutations at positions not previously linked with NNRTI resistance: 102, 139, 219, 241, 376 and 382 was confirmed by phenotyping site-directed mutants.

Conclusions: We successfully identified and validated novel NNRTI resistance associated mutations by developing parsimonious resistance prediction models in which repeated cross-validation within the stepwise regression was applied. Our model selection technique is computationally feasible for large data sets and provides an approach to the continued identification of resistance-causing mutations.
\end{abstract}

\section{Background}

Linear regression models have been shown to be accurate in predicting drug susceptibility from the HIV-1 viral genotype, by calculating the inhibitory concentration $50 \%\left(\mathrm{IC}_{50}\right) \log$ Fold-Change $(\mathrm{FC})$ phenotype as a linear combination of parameters, which are mutations [1-3] and interaction terms (mutation pairs) [1]. The

\footnotetext{
* Correspondence: kvdborgh@its.jnj.com

${ }^{1}$ Tibotec-Virco, Beerse, Belgium

Full list of author information is available at the end of the article
}

coefficients of these parameters are named resistance weight factors (RWF), and they quantify the effect on the $\log \mathrm{FC}$ of the mutations and mutation pairs. To generate models that are able to make predictions for future genotypes, ideally only resistance associated mutations are selected for the models. As it is not feasible to explore all possible subsets of mutations, stepwise regression is used to incrementally generate a series of regression models by addition or removal of mutations in each step. Different performance criteria exist to select one final linear regression model from this series

\section{() BiolMed Central}


[4,5]. In [1] standard stepwise regression was applied, selecting mutations based on significance with the F-test using predefined p-values. However, since correction for multiple significance-testing is not taken into account and because p-value thresholds are arbitrary, other selection criteria are preferred. Information criteria exist that balance accuracy and parsimony by penalizing for the number of parameters in the models. Information criteria commonly used are Akaike Information Criterion (AIC [6]) and Schwarz Bayesian Criterion (SBC [7]) with the penalty in SBC being more severe than in AIC. Although standard stepwise regression is a fast method in generating a model, the model found may be too complex by containing redundant information. Therefore, techniques are required that increase the stability of subset selection in linear regression. In [8] bootstrap aggregation ('bagging') was presented where an averaged prediction is made using multiple models generated on random re-samples of the original data set with replacement ('bootstrap') [9]. In [10] the bootstrap was integrated in the automatic selection procedure itself as parameters were sequentially added according to the proportion of bootstrap models in which they were selected. We investigated whether cross-validation [4,5] could be used as a less computationally intensive resampling technique than bootstrapping to reduce the complexity of the linear regression models while maintaining accuracy and adding to interpretability by generating only one regression model. As such, we aimed for an improvement in reliability of information extracted from the models, in this case the identification of novel mutations that cause resistance to anti-HIV drugs. In this article we use VirtualPhenotype ${ }^{\mathrm{TM}}$-LM (Virco, Beerse, Belgium) [1] as a reference prediction model. Since June 2006, VirtualPhenotype ${ }^{\mathrm{TM}}-\mathrm{LM}$ has been a linear regression model that predicts the log FC based on mutations (first-order terms) and mutation pairs (second-order interaction terms accounting for synergistic and antagonistic effects). We propose a more robust selection procedure by making two major changes to this reference approach. First, models were developed directly in second-order: interaction terms could be selected as soon as the constituting mutations were both present as first-order effect. Second, mutations or interaction terms were selected by repeatedly applying 3-fold cross-validation. In this article we refer to this method as $3 \mathrm{~F}$. Repeated cross-validation was presented as a way to reduce variability in the prediction error estimate [11]. Moreover, as shown in [12,13], repeated multi-fold cross-validation leads to better model selection when increasing the size of the validation set. To evaluate the generalizability of the models, the prediction error was calculated on genotypes in an unseen data set with available measured phenotypes.
After generating linear models with reduced complexity we were able to effectively identify novel mutations associated with NNRTI resistance. The individual contribution to resistance of these mutations was experimentally validated by making site-directed mutants and determining in vitro resistance levels.

\section{Results}

\section{Reverse Transcriptase Inhibitors}

For the reverse transcriptase inhibitors (RTI) a 3F model with lower complexity (using less mutations and less interaction terms) than the reference was found for AZT, 3TC, d4T, ABC, FTC, NVP, EFV and ETR (Table 1 ). For the nucleoside reverse transcriptase inhibitors (NRTI) class of drugs the reduction in interaction terms and mutations used in 3F versus reference was $20.3 \%$ and $11.9 \%$, respectively. For the NNRTI class of drugs the $3 \mathrm{~F}$ method was even more effective in reducing the complexity: the reduction in interaction terms and mutations was $38.3 \%$ and $26 \%$, respectively. For all RTI with exception of AZT the 3F performance on unseen data equalled the reference (3F average squared error within $1 \%$ of the reference) or was better than the reference (for ETR: 3F average squared error 1.9\% lower than in the reference) (Table 1). Moreover, the 3F model performance as compared to reference was maintained in subsets of unseen data samples, including the subset with one or more mutations included only in the reference model. Although the AZT 3F model had a lower SBC value on the training set than the reference, the averaged squared error on the unseen data was $1.5 \%$ higher in 3F. The reduction in the AZT 3F model compared to the reference of $28.7 \%$ interaction terms and $17.1 \%$ mutations thus resulted in $3 \mathrm{~F}$ underfitting. Nevertheless, for AZT the concordance in susceptibility calls on the unseen data between $3 \mathrm{~F}$ and reference was 94.67\% and there were no 'major' discordances (fully susceptible or maximal response by reference but fully resistant or minimal response by $3 \mathrm{~F}$ or vice versa) between the two approaches. In contrast, the ETR reference model had a lower SBC value on the training set than the 3F model but used 2.2 times more interaction terms and 1.3 times more mutations than the $3 \mathrm{~F}$ model, thus implying reference overfitting. Here, the concordance in susceptibility calls between the two approaches was $90.56 \%$, with one major discordance (see additional file 1: Comparison of susceptibility call between $3 \mathrm{~F}$ and Reference on unseen data for ATV, AZT and ETR). An increase in the ratio of interaction terms versus single terms in a 3F RT inhibitor model had no significant impact on the 3F performance compared with the reference $(P=0.3673)$. In total, 172 and 196 different mutations were used as single terms in the NRTI and NNRTI 3F models, respectively (Figure 1, and see 
Table 1 Complexity and performance of 3F and Reference models on genotype-phenotype data sequenced at Virco up to September 2006

\begin{tabular}{|c|c|c|c|c|c|c|c|c|c|c|}
\hline \multirow[b]{3}{*}{ drug } & \multirow[b]{3}{*}{$\begin{array}{c}N \\
\text { train }\end{array}$} & \multirow{2}{*}{\multicolumn{3}{|c|}{ Reference Sep 2006}} & \multirow{2}{*}{\multicolumn{3}{|c|}{$3 F$ Sep $2006^{a}$}} & \multirow{2}{*}{\multicolumn{3}{|c|}{$\begin{array}{c}\text { Unseen data } \\
\text { Sep } 2006 \text { - Dec } 2008\end{array}$}} \\
\hline & & & & & & & & & & \\
\hline & & $\begin{array}{l}\text { single }^{b} \\
\text { terms }\end{array}$ & $\begin{array}{c}\text { int }^{\mathrm{c}} \\
\text { terms }\end{array}$ & $\mathrm{mut}^{\mathrm{d}}$ & $\begin{array}{l}\text { single } \\
\text { terms }\end{array}$ & $\begin{array}{c}\text { int } \\
\text { terms }\end{array}$ & mut & $\begin{array}{c}N \\
\text { test }\end{array}$ & $\begin{array}{c}\text { ase }^{\mathrm{e}} \\
\text { Reference }\end{array}$ & $\begin{array}{c}\text { ase } \\
3 \mathrm{~F}\end{array}$ \\
\hline \multicolumn{11}{|c|}{ Nucleoside RT inhibitors } \\
\hline AZT & 45734 & 80 & 108 & 123 & 66 & 77 & 102 & 8698 & 0.091 & 0.093 \\
\hline 3TC & 47422 & 59 & 64 & 70 & 43 & 52 & 45 & 8733 & 0.059 & 0.059 \\
\hline ddl & 47269 & 49 & 21 & 62 & 50 & 25 & 54 & 8746 & 0.054 & 0.054 \\
\hline$d 4 T$ & 47235 & 47 & 34 & 68 & 54 & 20 & 60 & 8749 & 0.050 & 0.050 \\
\hline$A B C$ & 45908 & 71 & 46 & 90 & 63 & 24 & 68 & 8749 & 0.048 & 0.048 \\
\hline FTC & 16440 & 31 & 35 & 46 & 34 & 34 & 36 & 8722 & 0.086 & 0.086 \\
\hline TDF & 31640 & 64 & 91 & 110 & 79 & 83 & 111 & 8757 & 0.065 & 0.064 \\
\hline \multicolumn{11}{|c|}{ Nonnucleoside RT inhibitors } \\
\hline NVP & 47400 & 124 & 190 & 142 & 103 & 148 & 110 & 8729 & 0.101 & 0.100 \\
\hline EFV & 46054 & 191 & 167 & 211 & 126 & 101 & 142 & 8687 & 0.266 & 0.264 \\
\hline ETR & 18166 & 122 & 158 & 160 & 94 & 72 & 119 & 8493 & 0.126 & 0.124 \\
\hline
\end{tabular}

a July-September genotype-phenotype 2006 data was used as validation set for $3 \mathrm{~F}$.

${ }^{\mathrm{b}}$ Number of single terms (first order effects) in model.

${ }^{\mathrm{c}}$ Number of interaction terms in model.

${ }^{\mathrm{d}}$ Number of mutations in model.

${ }^{\mathrm{e}}$ Average squared error on unseen genotype-phenotype data collected between September 2006 and December 2008

additional file 2: Complexity and performance of $3 \mathrm{~F}$ and Reference models on genotype-phenotype data sequenced at Virco up to September 2006). The latter

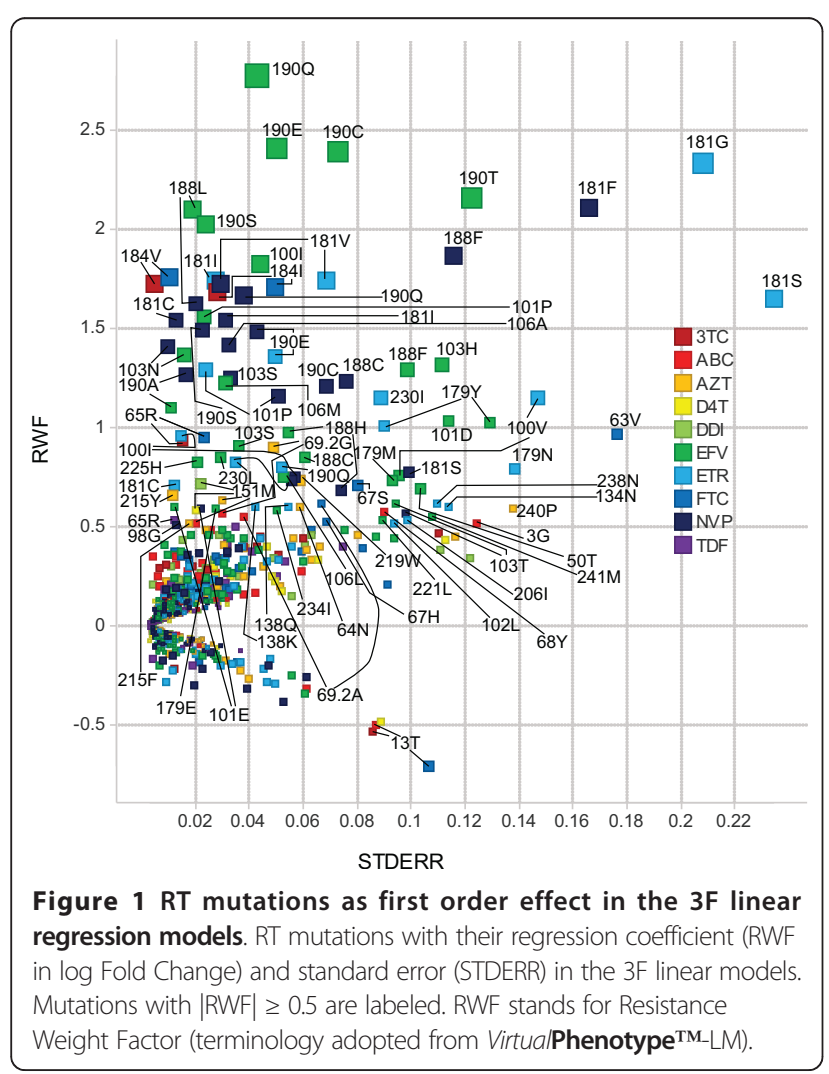

include all of the 44 NNRTI resistance-associated mutations identified in [14], with the exception of 179G, 227C, and 236L. Seventy-two RT mutations were present as single terms in both NRTI and NNRTI 3F models, including the highly prevalent mutations $103 \mathrm{~N}$ and $184 \mathrm{~V}$. Three interaction terms were common between the AZT 3F model and one or more of the NNRTI 3F models: 103N\&181C, 103N\&184V and 215Y\&219E. Some rare variants at RT position 181 were identified with high RWF and relatively high standard error: 181F, $181 \mathrm{G}$ and $181 \mathrm{~S}$ (Figure 1).

Linear discriminant analysis (LDA $[5,15]$ ) was conducted to compare the predicted FC distribution of genotypes with a mutation from the list compiled in [14] to the genotypes having the wild-type amino acid at the corresponding position. Mutations with the highest impact (LDA F1 [16]) on NNRTI resistance are shown in Figure 2. Ranking by impact (LDA F1) was largely similar when comparing the 3F models to the reference, and the RWF were also very similar in both approaches. However, some clear differences between 3F and reference were observed. For example, for EFV, 190Q (the mutation with the highest RWF in reference and 3F) had more impact in $3 \mathrm{~F}(\mathrm{~F} 1=0.133$ vs. 0.089). For ETR, mutation $179 \mathrm{~F}$ had more impact in $3 \mathrm{~F}$ than in the reference, although the mutation was not present as firstorder effect in the 3F model (Figure 2). To detect novel NNRTI resistance-associated mutations a similar LDA was conducted for the remaining $100 \mathrm{RT}$ mutations from the list of 124 found as first-order effect in the 


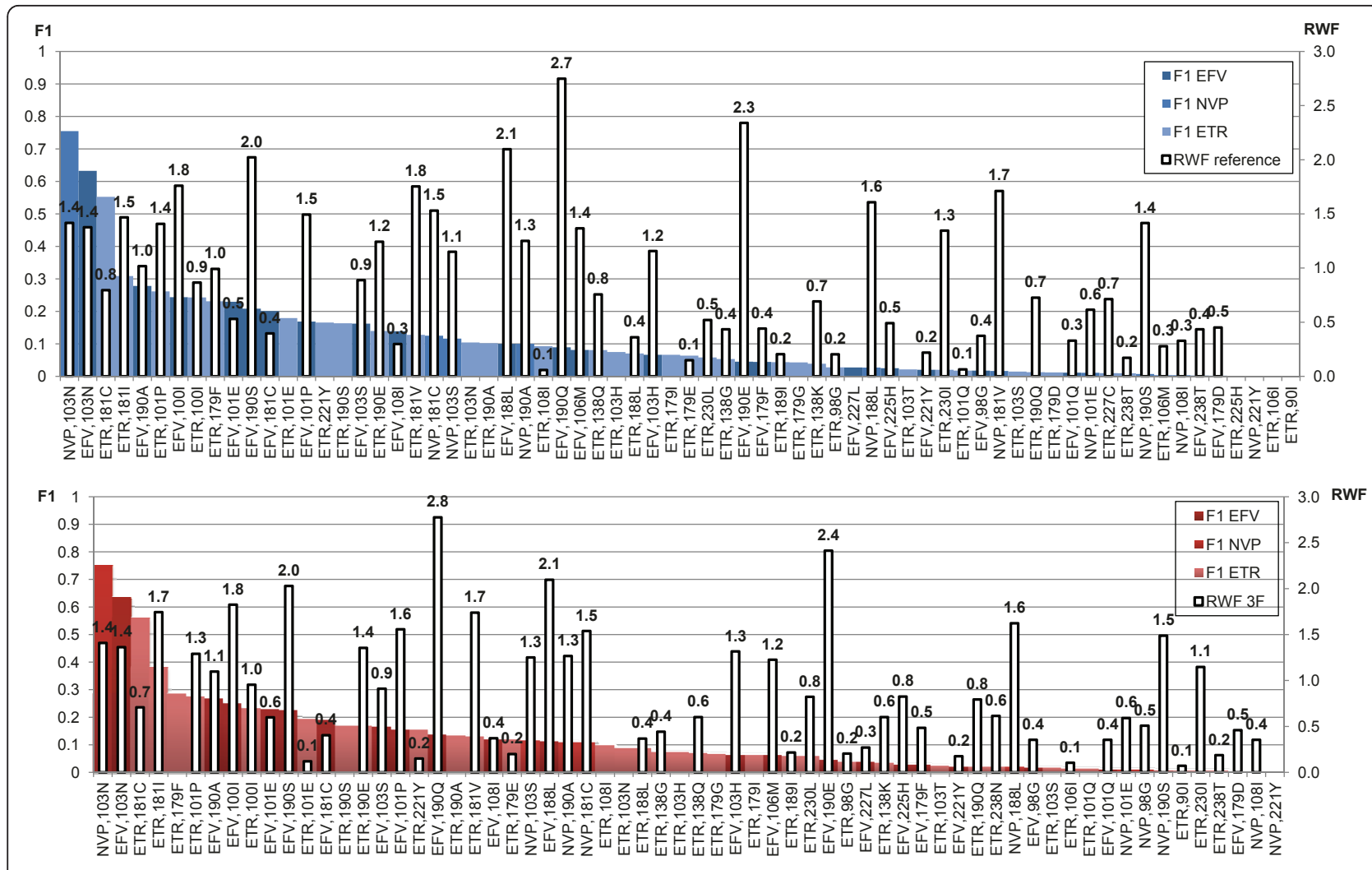

Figure 2 Impact of known NNRTI resistance associated mutations. LDA on reference (blue) and 3F (red) predicted phenotypes. Mutations shown are from the list of 44 known non-nucleoside RT inhibitor resistance associated mutations [14] having F1 > 0, ranked by F1.

NNRTI 3F models but not in the NRTI 3F models. We ranked these novel mutations by their impact on NNRTI resistance, and retained the top 40 for further analysis as described in the next section.

\section{Novel resistance-associated mutations}

Forty novel NNRTI resistance-associated mutations were found satisfying the following criteria: LDA cutoff $>0$ (mutation contributes to resistance) for all of the NNRTIs, RWF $>0$ in the $3 \mathrm{~F}$ model of at least one of the NNRTIs, and RWF $\nless 0$ in any 3F model of NVP, EFV or ETR (Table 2). The mutation with the highest impact (LDA F1) was $179 \mathrm{M}$ and occurred relatively rarely (31 times) in the LDA data set containing approximately 79,000 genotypes. Novel mutations that were more frequent with an impact $>0.01$ were $376 \mathrm{~S}$, 138A and 357T. Mutation 138A has recently been recognized as an ETR resistance associated mutation [17]. Site-directed mutants were made for a selection of the novel mutations. Their resistance contribution was analyzed by looking at the mutant's FC relative to a drug-specific biological cutoff (BCO), used to separate viruses that are susceptible from those that show signs of resistance [18]. When comparing ETR to the other two NNRTIs in Table 2 in most cases the LDA F1 value for ETR was higher than for EFV and NVP (Table 2: $\max (\mathrm{F} 1))$. However, the in vitro effect of the site-directed single mutations was, with exception of $138 \mathrm{~A}$, always below the BCO for ETR, while this was not the case for EFV and NVP. SDMs 100V, 190T, 101A and $101 \mathrm{D}$ had measured FC values above the BCO for NVP (> 6.0) and EFV (> 3.3). SDM 139R had an FC value above the BCO for NVP only. For the SDM 138A, the highest FC values measured were 4.9, (NVP, below $\mathrm{BCO}), 3.6$, (EFV, above BCO) and 3.5, (ETR, above BCO (> 3.2)). Other mutations found with elevated FC for at least one of the NNRTIs were 221L, 219H, 219D, 376S, 102L, 101N, 234I, 382T, 139K and 241M.

As these novel mutations frequently co-occur with known resistance-associated mutations, we also studied their effect in genetic background containing such mutations. The Virco database of clinical isolates was searched for the most appropriate genetic backgrounds to test each of the novel mutations for their effect on NNRTI resistance [19]. For the mutations 139R, 219D and $219 \mathrm{H}$ we looked at their contributions to resistance in combination with the highest-impact NNRTI resistance mutants $103 \mathrm{~N}$ and $181 \mathrm{C}$ (Figure 2, and see additional file 3: Linear Discriminant Analysis (LDA) for $103 \mathrm{~N}$ and $181 \mathrm{C})$. The following site-directed mutants 
Table 2 Novel non-nucleoside RT inhibitor resistance associated mutations

\begin{tabular}{|c|c|c|c|c|c|c|c|c|c|}
\hline \multirow[b]{2}{*}{ Mutation $^{\mathrm{c}}$} & \multirow[b]{2}{*}{$N w t^{\mathrm{d}}$} & \multirow[b]{2}{*}{$N m^{2} t^{\mathrm{e}}$} & \multicolumn{3}{|c|}{ Site-directed Mutants ${ }^{a}$} & \multicolumn{4}{|c|}{ LDA $3 F^{b}$} \\
\hline & & & NVP & EFV & ETR & $\max (F 1)$ & $\max \left(n_{11}\right)^{f}$ & $\max \left(\frac{n_{11}}{n_{11}+n_{01}}\right)^{8}$ & $\min (\text { cutoff })^{h}$ \\
\hline V179M & 67699 & 31 & $0.8-2.3$ & $0.3-0.4$ & $0.4-0.9$ & $0.327(\mathrm{EFV})$ & 13 (ETR) & $8 /(8+10)(E F V)$ & 1.35 (ETR) \\
\hline $\mathrm{H} 221 \mathrm{~L}$ & 76783 & 31 & $4.2-5.0$ & $1.5-1.6$ & 1.1 & 0.118 (EFV) & $2(\mathrm{EFV})$ & $2 /(2+1)(E F V)$ & 2.03 (ETR) \\
\hline V179Y & 67699 & 17 & $0.2-0.5$ & $<0.2-0.2$ & $<0.1-0.2$ & 0.063 (ETR) & 16 (ETR) & $16 /(16+472)(E T R)$ & 1.44 (ETR) \\
\hline $\mathrm{K} 219 \mathrm{H}$ & 67913 & 129 & $1.3-2.2$ & $1.0-1.8$ & $1.1-1.8$ & 0.047 (ETR) & 14 (ETR) & $14 /(14+447)(E T R)$ & 1.45 (ETR) \\
\hline K219D & 67913 & 161 & $1.4-3.8$ & $1.3-2.2$ & $0.4-2.0$ & 0.044 (ETR) & 24 (ETR) & $24 /(24+894)(E T R)$ & 1.22 (ETR) \\
\hline V179N & 67699 & 15 & $1.2-1.9$ & $0.9-1.2$ & $0.6-0.8$ & 0.039 (ETR) & 8 (ETR) & 8/(8 + 391) (ETR) & 1.50 (ETR) \\
\hline T376S & 15617 & 7415 & $2.8-3.4$ & $1.5-2.0$ & $1.6-2.1$ & 0.035 (EFV) & 135 (EFV) & $135 /(135+145)(E F V)$ & 1.67 (ETR) \\
\hline Y181S & 72643 & 3 & NA & NA & NA & 0.019 (ETR) & 3 (ETR) & $3 /(3+310)(E T R)$ & 1.45 (ETR) \\
\hline L100V & 77197 & 8 & $6.4(1)$ & $9.0-18.3(2)$ & $1.1-1.2(2)$ & 0.017 (ETR) & 6 (ETR) & $6 /(6+678)(E T R)$ & 1.63 (ETR) \\
\hline Y181F & 72643 & 9 & $1.1-2.0$ & $0.4-0.8$ & $0.4-0.7$ & 0.016 (ETR) & 4 (ETR) & $4 /(4+503)(E T R)$ & 1.33 (ETR) \\
\hline K102L & 72791 & 12 & $2.3-2.9$ & $0.5-1.2$ & $0.3-0.4$ & 0.015 (ETR) & 4 (ETR) & $4 /(4+505)(E T R)$ & 1.77 (ETR) \\
\hline K101N & 72175 & 94 & $5.0(1)$ & $2.8(1)$ & $0.8(1)$ & 0.015 (ETR) & 4 (ETR) & $4 /(4+434)(E T R)$ & 1.66 (ETR) \\
\hline V106L & 75529 & 44 & $0.5-0.6$ & $0.7-1.1$ & $0.2-0.3$ & 0.015 (ETR) & 5 (ETR) & $5 /(5+617)(E T R)$ & 1.69 (ETR) \\
\hline E138A & 75869 & 1828 & $2.2-4.9$ & $1.2-3.6$ & $2.8-3.5$ & 0.014 (ETR) & 14 (ETR) & $14 /(14+157)(E T R)$ & 2.10 (ETR) \\
\hline M357T & 44115 & 17866 & $0.9(1)$ & $1.2(1)$ & NA & 0.013 (ETR) & 117 (ETR) & $117 /(117+199)($ ETR) & 1.88 (ETR) \\
\hline T139R & 76899 & 243 & $6.4-7.3$ & $1.5-2.7$ & $1.0-1.2$ & 0.010 (ETR) & 2 (ETR) & $2 /(2+137)(E T R)$ & 2.22 (ETR) \\
\hline E370G & 75915 & 489 & NA & NA & NA & 0.007 (ETR) & 2 (ETR) & $1 /(1+1)(E F V)$ & 2.38 (ETR) \\
\hline I135T & 45829 & 18410 & NA & NA & NA & 0.006 (ETR) & 52 (ETR) & $52 /(52+94)(E T R)$ & 2.16 (ETR) \\
\hline L234I & 79037 & 98 & $0.6-1.0$ & $1.6-2.3$ & $0.9-1.1$ & 0.004 (ETR) & 1 (ETR) & $1 /(1+353)(E T R)$ & 1.95 (ETR) \\
\hline S379C & 69973 & 3578 & NA & NA & NA & 0.001 (ETR) & 1 (ETR) & $1 /(1+1)(E T R)$ & 3.35 (ETR) \\
\hline R206l & 79051 & 8 & $1.0-1.7$ & $0.4-0.7$ & $0.5-0.9$ & NA & 0 (ETR) & $0 /(0+102)(E T R)$ & 2.33 (ETR) \\
\hline S134N & 79041 & 19 & $1.2-2.1$ & $0.6-0.7$ & $0.8-0.9$ & NA & 0 (ETR) & $0 /(0+69)(E T R)$ & 2.45 (ETR) \\
\hline $\mathrm{H} 221 \mathrm{C}$ & 76783 & 59 & NA & NA & NA & NA & 0 (ETR) & $0 /(0+20)(E T R)$ & 2.73 (ETR) \\
\hline 1382T & 78025 & 329 & $2.4-6.7$ & $0.9-1.7$ & $0.7-2.4$ & NA & 0 (ETR) & $0 /(0+2)(E T R)$ & 3.30 (ETR) \\
\hline D237E & 78246 & 423 & NA & NA & NA & NA & $0(\mathrm{EFV})$ & $0 /(0+3)(E F V)$ & 3.67 (ETR) \\
\hline N348T & 74372 & 170 & NA & NA & NA & NA & 0 (ETR) & $0 /(0+0)(E T R)$ & 4.05 (ETR) \\
\hline E399G & 66049 & 670 & NA & NA & NA & NA & 0 (ETR) & $0 /(0+0)(E T R)$ & 4.10 (ETR) \\
\hline G190T & 72912 & 10 & $>67.4$ & $7.8-14.9$ & $0.6-0.7$ & NA & 0 (EFV) & $0 /(0+2)(E F V)$ & 4.16 (ETR) \\
\hline Y188F & 76892 & 41 & $1.4-1.9$ & $0.3-0.5$ & $0.2-0.6$ & NA & 0 (NVP) & $0 /(0+0)(N V P)$ & 4.70 (NVP) \\
\hline L283I & 72462 & 5930 & NA & NA & NA & NA & 0 (ETR) & $0 /(0+0)(E T R)$ & 5.01 (ETR) \\
\hline K101A & 72175 & 50 & 8.8-13.4 & $4.1-5.6$ & $1.5-1.8$ & NA & 0 (EFV) & $0 /(0+3)(E F V)$ & 5.04 (NVP) \\
\hline K101D & 72175 & 7 & $13.3-18.9$ & $5.7-6.8$ & $1.0-1.3$ & NA & 0 (EFV) & $0 /(0+3)(E F V)$ & 5.08 (EFV) \\
\hline T139K & 76899 & 348 & $4.4-5.8$ & $1.2-2.3$ & $2.4-3.0$ & NA & 0 (ETR) & $0 /(0+0)(E T R)$ & 5.10 (ETR) \\
\hline T165L & 75078 & 183 & NA & NA & NA & NA & 0 (ETR) & $0 /(0+0)(E T R)$ & 5.81 (ETR) \\
\hline T386A & 59810 & 1756 & NA & $1.6(1)$ & $0.5(1)$ & NA & 0 (NVP) & $0 /(0+0)(N V P)$ & 6.07 (NVP) \\
\hline V241M & 78771 & 23 & $4.7-5.6$ & $1.0-1.8$ & $0.8-1.2$ & NA & 0 (NVP) & $0 /(0+0)(N V P)$ & 6.90 (NVP) \\
\hline 1382L & 78025 & 228 & NA & NA & NA & NA & 0 (NVP) & $0 /(0+0)(N V P)$ & 7.30 (NVP) \\
\hline G335S & 65035 & 1877 & NA & NA & NA & NA & 0 (ETR) & $0 /(0+0)(E T R)$ & 7.55 (ETR) \\
\hline E399D & 66049 & 10697 & NA & NA & NA & NA & 0 (ETR) & $0 /(0+0)(E T R)$ & 7.98 (ETR) \\
\hline R358K & 70517 & 5995 & NA & NA & NA & NA & 0 (NVP) & $0 /(0+0)(N V P)$ & 8.06 (NVP) \\
\hline
\end{tabular}

${ }^{\mathrm{a}}$ Fold-Change range from 3 measurements, unless otherwise indicated between brackets.

FC > Biological Cut-Off (BCO) in bold, FC $\leq$ BCO in italic; BCO for NVP is 6.0, BCO for EFV is 3.3 and BCO for ETR is 3.2 .

${ }^{b}$ Summarized for the three non-nucleoside RT inhibitors (NNRTI).

${ }^{\mathrm{C}}$ Top 40 mutations, ranked by $\max (\mathrm{LDA} F 1)$ descending, then by $\min$ (LDA cutoff) ascending. Mutations shown are from the list of 124 NNRTI mutations with RWF $\geq 0$ and LDA cutoff $>0$ for NVP, EFV and ETR. Known NNRTI positions or novel mutations listed in [32,33] are shown in bold.

${ }^{\mathrm{d}}$ Frequency of wild-type (not within a mixture) in LDA data set.

e Frequency of mutation (not within a mixture) in LDA data set.

${ }^{f} n_{11}$ is the number of samples with amino acid mutation having a predicted phenotype above the LDA cutoff.

${ }^{g} n_{01}$ is the number of samples with wild type amino acid having a predicted phenotype above the LDA cutoff.

${ }^{h}$ Cutoff in log Fold-Change (taking the wild-type and mutation frequency percentages as prior probabilities in the LDA can result in cutoff values outside of the range of the predicted phenotypes). 
were tested for all NNRTIs: $103 \mathrm{~N}, 103 \mathrm{~N}+181 \mathrm{C}, 139 \mathrm{R}$ $+103 \mathrm{~N}+181 \mathrm{C}, 219 \mathrm{D}+103 \mathrm{~N}+181 \mathrm{C}$ and $219 \mathrm{H}+103 \mathrm{~N}+181 \mathrm{C}$ (Table 3). While for NVP and EFV all of the above combinations were resistant, for ETR the single mutation $103 \mathrm{~N}$ and the combination $103 \mathrm{~N}+181 \mathrm{C}$ were susceptible with mean FC values of 0.9 and 3.0, respectively. The limited impact (3F LDA F1: 0.09) of $103 \mathrm{~N}$ on ETR resistance was thus confirmed by the site-directed mutant and adding $181 \mathrm{C}$ did not result in a FC above the BCO. Remarkably, all the above triple combinations were found to be resistant for ETR, thereby clearly demonstrating the contribution to NNRTI resistance of the novel mutations. For ETR a 2.2, 6.3 and 4.6-fold increase in FC compared to $103 \mathrm{~N}+181 \mathrm{C}$ was seen when adding 139R, 219D and 219H, respectively. For EFV a 1.8-2.6fold increase was seen as well. For NVP the contribution to resistance of the novel mutations in combination with $103 \mathrm{~N}+181 \mathrm{C}$ could not be confirmed due to $\mathrm{IC}_{50}$ values larger than the maximum assay concentration (Table 3).

Table 3 Site-Directed Mutants of novel NNRTI resistance associated mutations 139R, 219D and 219H in combination with $103 \mathrm{~N}+181 \mathrm{C}$ and SDM $181 \mathrm{G}$

\begin{tabular}{|c|c|c|c|c|}
\hline \multirow{2}{*}{$\frac{\text { SDM }}{139 \mathrm{R}}$} & \multirow{2}{*}{$\begin{array}{c}\text { drug } \\
N V P\end{array}$} & \multicolumn{3}{|c|}{3 measurements (Fold Change ${ }^{a}$ ) } \\
\hline & & 7.3 & 7.3 & 6.4 \\
\hline & EFV & 1.5 & 2.2 & 2.7 \\
\hline & ETR & 1.2 & 1.2 & 1.0 \\
\hline \multirow[t]{3}{*}{$219 D$} & NVP & 3.7 & 3.8 & 1.4 \\
\hline & EFV & 1.3 & 2.2 & 1.3 \\
\hline & ETR & 1.1 & 2.0 & 0.4 \\
\hline \multirow[t]{3}{*}{$219 \mathrm{H}$} & NVP & 2.1 & 2.2 & 1.3 \\
\hline & EFV & 1.1 & 1.0 & 1.8 \\
\hline & ETR & 1.8 & 1.7 & 1.1 \\
\hline \multirow[t]{3}{*}{$103 \mathrm{~N}$} & NVP & $>51.7$ & $>54.2$ & 35.4 \\
\hline & EFV & 19.5 & 15.1 & 12.5 \\
\hline & ETR & 1.1 & 0.9 & 0.6 \\
\hline \multirow[t]{3}{*}{$103 N+181 C$} & NVP & $>85.9$ & $>85.9$ & $>85.9$ \\
\hline & EFV & 28.4 & 23.1 & 38.7 \\
\hline & ETR & 4.2 & 1.7 & 3.2 \\
\hline \multirow[t]{3}{*}{$139 R+103 N+181 C$} & NVP & $>22.7$ & $>21.8$ & $>22.7$ \\
\hline & EFV & 60.2 & 77.0 & 61.2 \\
\hline & ETR & 7.0 & 7.4 & 5.6 \\
\hline \multirow[t]{3}{*}{$219 D+103 N+181 C$} & NVP & $>79.6$ & $>79.6$ & $>76.7$ \\
\hline & EFV & 116.6 & 72.9 & 48.6 \\
\hline & ETR & 15.5 & 20.2 & 21.4 \\
\hline \multirow[t]{3}{*}{$219 \mathrm{H}+103 \mathrm{~N}+181 \mathrm{C}$} & NVP & $>79.6$ & $>79.6$ & $>79.6$ \\
\hline & EFV & 92.6 & 23.7 & 49.0 \\
\hline & ETR & 14.5 & 16.1 & 11.6 \\
\hline \multirow[t]{3}{*}{$181 \mathrm{G}$} & NVP & 30.1 & 38.3 & $>37.2$ \\
\hline & EFV & 1.6 & 1.3 & 1.1 \\
\hline & ETR & 1.5 & 0.9 & 1.2 \\
\hline
\end{tabular}

a > Biological Cut-Off (BCO) in bold, $\leq$ BCO in italic; BCO for NVP is 6.0, BCO for EFV is 3.3 and BCO for ETR is 3.2.
Data for the novel mutations 102L, 138A, 139K, 139R, $179 \mathrm{Y}, 181 \mathrm{~F}, 188 \mathrm{~F}, 221 \mathrm{~L}$ and $234 \mathrm{I}$ tested in different genetic backgrounds can be found in additional file 4: Site directed mutants of novel mutations tested for NVP, EFV and ETR, extending Table 2 with results of combinations.

Mutation 181G was not in the list of novel mutations as it was always found in presence of other amino acids at position 181 (a 'mixture') in the LDA data set. A sitedirected mutant, containing $181 \mathrm{G}$ only, was made and found to be resistant to NVP (FC: 30.1-38.3) and having FC values of 1.1-1.6 for EFV and 0.9-1.5 for ETR (Table 3).

\section{Discussion}

In order to quantitatively predict drug resistance from the HIV-1 viral genotype we used 3-fold cross-validation for the stepwise selection of model mutations or mutation pairs. Importantly, we applied a random division in three parts before each removal step (see additional file 5: K Fold cross-validated stepwise regression using same or different random division before each removal step: ETR model). Thus, in the $3 \mathrm{~F}$ method robustness was achieved by removing model parameters that were not consistently selected under different random divisions. As a consequence, the $3 \mathrm{~F}$ method can be considered a greedy stepwise approach where the probability of a parameter being removed increases as the model size increases. As we had to generate models for several drugs this computational approach was a practical one for our purpose. Nevertheless, to make the model selection less greedy, one might consider repeating the crossvalidation at each step multiple times with a different random division in three parts, to improve the estimation of the prediction error.

As our goal was to reduce the complexity and maintain the accuracy of the reference models, we did not investigate defining a stop criterion for the $3 \mathrm{~F}$ method independent from the reference. Instead, we retained all $3 \mathrm{~F}$ models with better performance (AIC/SBC) than the reference on the genotype-phenotype data, holding out the two last months. The $3 \mathrm{~F}$ model with best performance on the hold-out set was then selected as the final model, and the 3F model parameters were recalculated on the genotype-phenotype data including the hold-out set. Ultimately, in deciding between different approaches, the evaluation of performance on future observations remains the first priority. Therefore we tested the performance of the reference and $3 \mathrm{~F}$ method on a large unseen genotype-phenotype data set sequenced at Virco between September 2006 and December 2008. For the RT inhibitors the 3F method maintained the accuracy of the reference models while reducing complexity of the linear regression models. 
We also evaluated the performance of the 3F method for protease inhibitors (see additional file 2: Complexity and performance of $3 \mathrm{~F}$ and Reference models on genotype-phenotype data sequenced at Virco up to September 2006). An equal performance between $3 F$ and reference was seen for the most recent protease inhibitors TPV and DRV, and with both methods the models were less complex than for the other PIs. For the older protease inhibitors the performance of the reference approach was better than the 3F approach. This suggests that protease inhibitors for which resistance patterns have become more complex over time also require more complex regression models, thus increasing the importance of interaction terms. This is in conflict with the constraint in the $3 \mathrm{~F}$ method that allowed only interaction terms of which both first order effects were already present in the model during the stepwise regression.

For the identification of resistance-associated mutations we restricted our attention to the NNRTIs. We detected novel variants at NNRTI resistance-associated positions 100,101, 179, 181, 188, 190, 221, as well as at novel NNRTI resistance-associated positions 102, 139, 219, 241, 376 and 382.

As genotyping was done up to RT amino acid (AA) position 400, the $3 \mathrm{~F}$ linear models of the RTIs, with exception of 3TC and FTC, also contained connection domain mutations (AA 289-423 of RT). Two out of eight of the connection domain mutations described in [20] to be associated with AZT resistance, were found to increase resistance as single term in the AZT 3F model: $348 \mathrm{I}$ and $360 \mathrm{~V}$. In [21], $376 \mathrm{~S}$ was found to be associated with an increased risk of virological failure to NVP-based therapy in NNRTI-naïve patients and we could confirm the in vitro effect on NNRTI resistance by site-directed mutagenesis. In [22], resistance predictions for AZT, NVP and EFV by current genotypic drug resistance interpretation systems were found to be noninferior when predicting from short RT sequences (AA 41-238 for RT) compared to full RT sequences. Nevertheless, our analysis suggests that there are 12 novel NNRTI resistance associated connection domain mutations, including $376 \mathrm{~S}$ and $382 \mathrm{~T}$, that could provide more accurate predictions of NNRTI drug resistance.

Mutations found as a single term in both NRTI and NNRTI 3F models consisted of mutations at NRTI positions associated with NNRTI hypersusceptibility [23-25], including $215 \mathrm{Y}$, found to be ETR hypersusceptible in the reference and $3 F$ models, of connection domain mutations, including 348I, and of NNRTI resistance associated mutations from the list compiled in [14]. Our 3F models suggest that these latter mutations might have an effect on NRTI resistance as well (two have already been confirmed to have such an effect: 100I [26] and $181 \mathrm{C}$ [27]). In this study we were interested in novel NNRTI resistance associated mutations and we considered RT mutations found in the NNRTI 3F models only.

By making site-directed mutants we compared the resistance effect of mutations as first-order effect in the models with the in vitro effect. These results show that despite the reduction in model complexity for the $3 \mathrm{~F}$ approach, one must still be cautious when equating linear model coefficients with in vitro effect. For example, mutation $181 \mathrm{~F}$ has only a small in vitro effect for NVP whereas in the $3 \mathrm{~F}$ regression model this mutation has a high first-order coefficient. A likely explanation for this is that in the same $3 \mathrm{~F}$ model interaction terms of $181 \mathrm{~F}$ with 98G, 103N, 106M, 190A (all known resistance key mutations [14]) with a strong predicted resensitizing effect are present as well, canceling out the resistance contribution of $181 \mathrm{~F}$ as first-order effect. Specifically, we could confirm the resensitizing effect of the combination $181 \mathrm{~F}+103 \mathrm{~N}$ by comparing the resistance levels of SDM $181 \mathrm{~F}+103 \mathrm{~N}$ and SDM 103N. (see additional file 4: Site directed mutants of novel mutations tested for NVP, EFV and ETR). The systematic co-occurrence of $181 \mathrm{~F}$ with known resistance mutations probably resulted in a high ranking for mutation $181 \mathrm{~F}$ in the list of novel NNRTI resistance associated mutations.

As another example for ETR $179 \mathrm{~F}$ is present as a firstorder effect with a large resistance coefficient in the reference model whereas in the $3 \mathrm{~F}$ model $179 \mathrm{~F}$ is not present as a first-order effect. Inspecting this further we found the interaction term $179 \mathrm{~F} \& 181 \mathrm{C}$ to be present with a strong resistance effect in the $3 \mathrm{~F}$ model, but absent in the reference model. In this case the 3F method was in line with in vitro selection experiments done in [28], where the synergistic effect of $181 \mathrm{C}$ and $179 \mathrm{~F}$ on the decreased susceptibility of ETR was already described. Moreover, the one genotype with measured resistant phenotype that resulted in a major discordance for ETR between 3F (Minimal Response) and reference (Maximal Response) contained exactly the 179 F\& $181 \mathrm{C}$ combination (see additional file 1: Comparison of susceptibility call between 3F and Reference on unseen data for ATV, AZT and ETR). Most of the novel derived NNRTI resistance associated mutations had the highest impact (LDA F1 value) on resistance for ETR but a relatively low in vitro effect in comparison with NVP and EFV. Co-occurrence of multiple resistance associated mutations is needed to cause an elevated ETR FC, as exemplified by the SDMs containing two known resistance associated mutations and one novel mutation (e.g. 139R $+103 \mathrm{~N}$ $+181 \mathrm{C}$ ) with FC values above the BCO for ETR.

\section{Conclusions}

By applying repeated 3-fold cross-validation within the stepwise regression, we could lower the complexity of 
linear regression models for predicting drug resistance while retaining performance on unseen data. The described 3F method thus proves to be a tractable approach when interpretation of the linear model is an objective. As the 3F method worked particularly well for the non-nucleoside reverse transcriptase inhibitors, we derived a list of forty novel NNRTI resistance associated mutations. For a selection of the novel mutations we confirmed their in vitro contribution to resistance by site-directed mutagenesis, individually or in combination with known resistance mutations. As most of these novel mutations were found at relative low frequency in patient samples, carefully following up on drug resistance in patients with viruses carrying these mutations may provide more insight in understanding how the virus escapes the current antiretroviral treatment, as well as in the design of novel drugs.

\section{Methods}

\section{Genotype-phenotype database}

The size of the data sets of genotypes (AA 1-99 of Protease (PR) and AA 1-400 of RT) with an available measured phenotype (Antivirogram, Virco) ranged from approximately 20,000 to 56,000 samples for all protease and reverse transcriptase inhibitors. Phenotypes were measured as the $\log \mathrm{FC}$ in $\mathrm{IC}_{50}$ of a sample relative to a wild-type laboratory reference strain.

\section{Reference Linear Models}

VirtualPhenotype ${ }^{\mathrm{TM}}$ Linear Models were calculated for all drugs on samples sequenced at Virco up to July 2006 and recalculated 2 months later (samples up to September 2006). Two stepwise regression procedures were used to develop the models [1]. First, a model was calculated using single mutations only. In the second stepwise regression procedure, all possible interaction terms containing the mutations present in the first-order model were also considered for selection, to account for synergistic and antagonistic effects. Cutoff values for p-values were used to determine which mutations or interaction terms could enter the model (SLE) or stay in the model (SLS) (Figure 3). The minimal number of occurrences in the database for mutations or interaction pairs to be considered in the model was 10 for FTC, ETR, ATV, TPV and DRV and 20 for all other drugs, limiting the number of mutations considered to approximately 300 for PR inhibitors and 1000 for RT inhibitors. Mutations occurring in mixtures (ambiguous sequencing results) were weighted accordingly, mixtures of more than four amino acids were not considered. No validation set was used in selection of the reference models.

\section{F Linear Models}

In the $3 \mathrm{~F}$ method three fold cross-validated prediction error sum of squares (CVPRESS) was used as selection

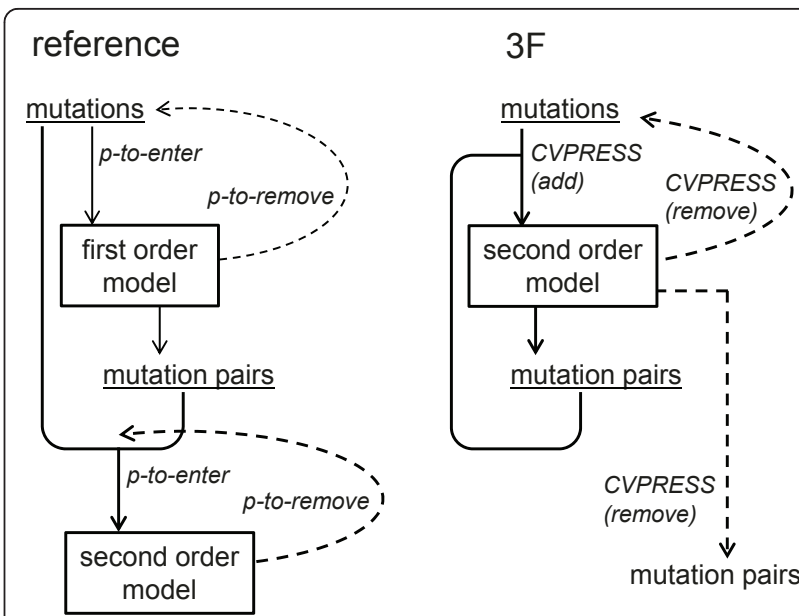

Figure 3 Reference and 3F methodology: schematic overview. In the $3 \mathrm{~F}$ method cross-validated prediction error (CVPRESS) was used instead of significance levels ( $p$-values) in the reference approach. In the reference approach two stepwise regression procedures were used: all possible mutation pairs were made from mutations in the first order model and candidate for entry in the second order model. In the 3F method, the initial search space consisted of all individual mutations. Mutation pairs could only enter the model if both mutations in the pair were already selected for the model.

criterion for parameter inclusion, instead of the significance levels (SLS and SLE) used in the reference approach. The motivation for this choice of fold is described in the next section. During 3-Fold cross-validation the data set is randomly split in three equal-sized parts, and a model is generated three times on two of the three parts and validated/tested on the remaining part. In this repetitive modeling each part is used only once for validation so that every genotype is treated once as unseen. A mutation was selected based on the performance (prediction error) calculated for the 'unseen' genotypes. We applied this cross-validation directly in each step of the selection procedure in evaluating which parameter to add or which parameter(s) to remove from the model. The initial search space consisted of all individual mutations. As soon as two mutations at different positions were selected, the cross-term of both could enter the model (we did not force this hierarchy constraint in the removal step, meaning that mutations could be removed as first-order effect while still present in an interaction term). The search space was thus dynamically enlarged with interaction terms as more single mutations were added (Figure 3). Models were calculated on the July 2006 data set. The same minimal counts $(10 / 20)$ as for building the July 2006 reference model were used as thresholds for mutations to enter the $3 \mathrm{~F}$ model. No minimal count was set for incorporation of interaction terms, as the $3 \mathrm{~F}$ method 
already in concept selected for less interaction terms. The CVPRESS selection criterion can be specified directly in the SAS GLMSELECT procedure [29]. Therefore CVPRESS can be applied in the same way as selection criteria like AIC or SBC.

\section{Randomized stepwise selection}

By using CVPRESS as selection criterion, a choice of fold $\mathrm{K}$ had to be made. We selected ETR as drug for evaluating different $\mathrm{K}$. In short, the results of this evaluation were as follows (see additional file 5: K Fold cross-validated stepwise regression using same or different random division before each removal step: ETR model). Keeping the same random division throughout the stepwise regression, $\mathrm{K}=\mathrm{N}$ (Leave-One-Out, also called PRESS [4]) was found too costly to compute $[5,30]$. Moreover, as the $\mathrm{N}$ training sets were almost similar, the improvement on the reference was rather a result of the hierarchy constraint for introduction of interaction terms than of the cross-validation itself. Nevertheless, choosing $\mathrm{K}<\mathrm{N}$ automatically leads to selection bias. For $\mathrm{K}=3$, an apparent decrease in the ratio of $\left(\mathrm{SBC}_{\mathrm{CV}}-\mathrm{SBC}\right)$ over the number of model parameters was observed as the model size increased, suggesting overfitting. Therefore, to generate better generalizing models, we decided to alter the random division before each removal step in the stepwise regression. Moreover, we now found $\mathrm{K}=3$ to be the best choice of fold in generating a model with lower SBC than the reference, and using the lowest number of parameters. Choosing $\mathrm{K}=2$, the required performance, set by the reference, could not be achieved using a limited number of random divisions, due to an increase of the number of parameter removals. Consequently, for $\mathrm{K}$ $=2$, also the difference between $\mathrm{SBC}_{\mathrm{CV}}$ and $\mathrm{SBC}$ was found to be the largest, indicating that parameter selection became more difficult when only half of the samples was used for training of the model.

The randomized stepwise selection procedure can now be described. An initial forward stepwise regression was executed until two mutations at different positions were present in the model. After that the stepwise selection procedure amounted to the execution of multiple backward-forward regression steps cycles, with each cycle consisting of a backward/removal step followed by a forward/addition step. To avoid overfitting, a different random division in three parts was used in each of these cycles. As a consequence of this randomization, it was possible that the latest parameter added in the forward step of cycle $i$ was directly removed in the backward step of cycle $i+1$, for instance when a model parameter was only present in one of the three parts of the new random division. This was in fact the reason why we decided to alter the random division before each backward step and not before each forward step. Crossvalidation thus penalized infrequent interaction terms to get/stay in the model but was nevertheless less stringent than applying a minimal count rule as was done in the reference method.

\section{F model selection}

The Virco genotype-phenotype data set between July and September 2006 was used as validation/test set. The 3F models were then selected as follows (Table 4): i) select all models with a better performance than the reference model on the training set with either AIC or SBC and ii) from the models selected in i), select the model that has the best performance (average squared error) on the test set. The selected 3F models were then refitted on all samples (including the validation set) to actually compare them to the September 2006 reference models.

AIC is defined as $n \ln (S S E / n)+2 p$. SBC is defined as $n \ln (S S E / n)+\ln (n) p$. In the above definitions $S S E$ is the Sum of Squared Errors, $n$ is the number of genotypesphenotypes and $p$ is the number of parameters in the linear regression model [4].

\section{Linear Discriminant Analysis}

To rank RT mutations by their impact on NNRTI resistance, a linear discriminant analysis (LDA) was done on approximately 79,000 predicted phenotypes calculated from genotypes sequenced at Virco between September 2006 and December 2008. Note that the LDA was done on the calculated phenotypes and not on the measured phenotypes in order to incorporate as many recent genotypes as possible, especially since some of the novel NNRTI mutations are infrequent in occurrence (Table $2)$. In order to analyze the impact of a mutation at position $i$ on resistance, a division of genotypes into two groups was made. The first group contained all genotypes containing the wild-type (HXB2) amino acid at position $i$ and the second group the genotypes with the amino acid mutation. Genotypes containing a mixture of amino acids at the mutated position were not considered. A contingency table was then made to analyze how well the two groups could be separated using the LDA cutoff applied on the calculated phenotypes. The following metrics were calculated on the two-by-two contingency table: precision, recall and F1. Precision is defined as $\frac{n_{11}}{n_{11}+n_{01}}$. Recall is defined as $n_{11} / N_{1} \cdot n_{11}$ is the number of samples with the amino acid mutation having a calculated phenotype above the LDA cutoff. $n_{01}$ is the number of samples with the wild-type amino acid having a calculated phenotype above the LDA cutoff. $N_{1}$ is the number of samples with the amino acid mutation. As precision (positive predictive value) of the 
Table 4 3F model selection on genotype-phenotype data up to September 2006

\begin{tabular}{|c|c|c|c|c|c|c|c|c|c|}
\hline \multirow[b]{2}{*}{ drug } & & \multicolumn{3}{|c|}{ 3F Model generation ${ }^{a}$} & \multicolumn{5}{|c|}{ 3F Model selection ${ }^{b}$} \\
\hline & & \# 3F Models & \# lower SBC & \# lower AIC & lower $\mathrm{SBC}^{\mathrm{C}}$ & lower $\mathrm{AIC}^{\mathrm{d}}$ & $N$ test & ase & $3 \mathrm{~F}$ model selected ${ }^{\mathrm{e}}$ \\
\hline \multirow[t]{7}{*}{ Nucleoside RT inhibitors $^{f}$} & AZT & 300 & 86 & 0 & yes & no & 800 & 0.103 & 296 \\
\hline & 3TC & 150 & 60 & 34 & yes & no & 807 & 0.037 & 99 \\
\hline & ddl & 150 & 20 & 70 & no & yes & 807 & 0.049 & 83 \\
\hline & $\mathrm{d} 4 \mathrm{~T}$ & 120 & 41 & 35 & yes & no & 806 & 0.040 & 81 \\
\hline & $A B C$ & 200 & 111 & 53 & yes & no & 807 & 0.038 & 95 \\
\hline & FTC & 80 & 28 & 22 & yes & yes & 804 & 0.071 & 76 \\
\hline & TDF & 400 & 66 & 196 & no & yes & 807 & 0.039 & 298 \\
\hline \multirow[t]{3}{*}{$\mathrm{NNRTI}^{9}$} & NVP & 400 & 93 & 0 & yes & no & 801 & 0.089 & 391 \\
\hline & EFV & 500 & 101 & 0 & yes & no & 807 & 0.246 & 386 \\
\hline & ETR & 700 & 49 & 0 & yes & no & 777 & 0.113 & 656 \\
\hline \multirow[t]{8}{*}{ Protease inhibitors } & IDV & 485 & 50 & 51 & yes & yes & 805 & 0.075 & 482 \\
\hline & NFV & 375 & 64 & 6 & yes & yes & 808 & 0.063 & 375 \\
\hline & SQV & 600 & 53 & 0 & yes & no & 807 & 0.092 & 575 \\
\hline & APV & 1000 & 0 & 656 & no & yes & 808 & 0.060 & 709 \\
\hline & LPV & 500 & 205 & 28 & yes & no & 807 & 0.157 & 319 \\
\hline & ATV & 1275 & 0 & 2 & no & yes & 805 & 0.117 & $1158^{h}$ \\
\hline & TPV & 1000 & 641 & 142 & yes & no & 806 & 0.059 & 428 \\
\hline & DRV & 1000 & 823 & 799 & yes & yes & 816 & 0.096 & 707 \\
\hline
\end{tabular}

${ }^{a}$ The number of $3 \mathrm{~F}$ models generated was arbitrary but taken large enough such that at least one $3 \mathrm{~F}$ model was found with a lower SBC or AIC than the reference on the genotype-phenotype data set up to July 2006.

${ }^{\mathrm{b}}$ From the remaining $3 \mathrm{~F}$ models with lower SBC or AIC than the reference, the 3F model was then selected with the lowest average squared error (ase) on an unseen genotype-phenotype data set collected between July and September 2006 (test set) containing approximately 800 samples.

${ }^{\mathrm{c}} \mathrm{SBC}$ of the selected $3 \mathrm{~F}$ model $<\mathrm{SBC}$ reference on the test set (yes/no).

${ }^{\mathrm{d}} \mathrm{AIC}$ of the selected $3 \mathrm{~F}$ model $<$ AIC reference on the test set (yes/no).

${ }^{\mathrm{e}}$ The number of different random divisions used in the stepwise regression in the selected $3 \mathrm{~F}$ model.

${ }^{f}$ For the nucleoside RT inhibitors the number of random divisions needed was less than 100, with exception of AZT and TDF.

${ }^{g}$ For the non-nucleoside RT inhibitors most random divisions were needed for ETR.

${ }^{\mathrm{h}}$ ATV was the only drug for which more than 1000 different random divisions were needed.

LDA discrimination in $\log$ FC of the mutated group from the wild type group at a position $i$ can be high, while recall (sensitivity) is low or vice versa, we used the F1 metric, trading off precision and recall, for the ranking of mutations for their impact on resistance. F1 is defined as $\frac{(2 \times p \times r)}{(p+r)}$ and thus equally weights precision $(p)$ and recall $(r)$. Ranking by impact on resistance (F1) was done for the known NNRTI resistance-associated mutations. For novel mutations, exclusively present as first-order effect in the 3F NNRTI linear regression models (thus absent in $3 \mathrm{~F}$ nucleoside reverse transcriptase linear regression models), ranking for being associated with resistance was done using $\mathrm{F} 1$ if $p$ $+r>0$ and by LDA cutoff otherwise. LDA analysis was done for both the reference and $3 \mathrm{~F}$ calculated phenotypes calculated using the September 2006 models.

\section{Site-Directed Mutants}

Site-directed mutants were created at Eurofins Medigenomix GmbH (Ebersberg, Germany) using the linear reaction method. In this method, the template DNA is linearly amplified using a mutagenesis-grade high-fidelity DNA polymerase which extends the mutagenic primers containing the desired mutation, incorporating the mutation of interest into the newly synthesized strands. The unique primer design allows replication of only the parental strand. Final treatment with Dpn I ensures the digestion of only dam-methylated parental strands. The resulting mutagenic strands were then transformed in ultracompetent cells and cultured on an agar plate. Single colonies were sequenced to ensure the availability of the correct mutation in the strand. A colony of a correct mutation containing strand was cultured and the purified plasmid shipped to Virco. Starting from this plasmid, the Protease - Reverse transcriptase region (AA 1-99 of PR and AA 1-400 of RT) was amplified and transfected into $293 \mathrm{~T}$ cells and recombined with the deletion backbone by homologous recombination [31]. The cultivated virus was then grown against a standard set of anti-HIV drugs.

\section{Additional material}

Additional file 1: Comparison of susceptibility call between $3 \mathrm{~F}$ and Reference on unseen data for ATV, AZT and ETR. For ATV, AZT and 
ETR a concordance analysis in susceptibility calls was done using vircoTYPE 4.2 clinical cutoffs $[34,35]$. AZT and ETR were the RTIs with difference in average squared error between $3 \mathrm{~F}$ and reference larger than $1.0 \%$. ATV was the drug for which most random divisions were needed to generate the $3 \mathrm{~F}$ model (Table 4), and one of the Pls with averaged squared error $>7 \%$ higher in $3 \mathrm{~F}$ than in the reference.

Additional file 2: Complexity and performance of $3 \mathrm{~F}$ and Reference models on genotype-phenotype data sequenced at Virco up to September 2006. Complexity of the 3 F models for the NRTIs, NNRTIs and Pls, and performance on training and test set. The 296 RT mutations found as single term in the RTI 3 F models are listed as i) single terms exclusively found in NRTI 3F models, ii) single terms exclusively found in NNRTI 3F models and iii) single terms found in both NRTI and NNRTI 3F models.

\section{Additional file 3: Linear Discriminant Analysis (LDA) for $103 \mathrm{~N}$ and}

181C. 3F LDA F1 impact on resistance of $103 \mathrm{~N}$ is largest for NVP: 0.75 , then for EFV: 0.63 and then for ETR: 0.09. 3F LDA F1 impact on resistance of $181 \mathrm{C}$ is largest for ETR: 0.56 , then for EFV: 0.19 and then for NVP: 0.11 . LDA cutoff (blue line) is shown to discriminate between samples with wild type at position 103/181 and samples with mutation 103N/181C for which the density histograms are shown. Frequency of wild type (not within a mixture) in LDA data set was 62,010 and 72,643 for positions 103 and 181, respectively. Frequency of mutation (not within a mixture) in LDA data set was 12,012 and 5043 for $103 \mathrm{~N}$ and 181C, respectively.

Additional file 4: Site Directed Mutants of novel mutations tested for NVP, EFV and ETR. Fold Change (FC) was calculated as the $I_{50}$ of the site-directed mutant divided by the $\mathrm{I}_{50}$ of a wild-type laboratory reference strain. All SDMs were measured three times (unless indicated otherwise) and FCs for each of the three measurements are shown. SDMs used as genetic background for evaluating the contribution to resistance of the novel mutations, are given at the top of the file. Noteworthy, the in vitro drug resistance interaction mechanism of the novel mutation and the known NNRTI resistance associated mutations was not always additive: $181 \mathrm{~F}$ contributed to resensitization to EFV of the $103 \mathrm{~N}$ mutated virus, $179 \mathrm{Y}$ contributed to resensitization to NVP and EFV of the 190A mutated virus.

Additional file 5: $\mathrm{K}$ Fold cross-validated stepwise regression using same or different random division before each removal step: ETR model. Different choices of fold $\mathrm{K}$ were evaluated for the ETR model.

The goal was to find a linear regression model with better SBC than the reference and at the same time using less parameters. (A) When keeping the same random division during the stepwise regression, selection bias resulted in more overfitting, when lowering $\mathrm{K}$. By altering the random division before each removal step, for $K=3$ the reference goal SBC was reached with the lowest number of parameters. (B) The difference between $S_{C C}$ CV and SBC (calculated as $n \operatorname{In}(C V P R E S S / S S E$ )) was found to be larger when lowering the number of folds $K$, in case a different random division was used before each removal step. ( $C$ ) When lowering $\mathrm{K}$, using a different random division before each removal step resulted in more parameter removals. Whereas for $\mathrm{K}=3$, a model with the required SBC was found using 700 backward-forward cycles, for $K=2$, the model size did not increase fast enough during the stepwise procedure as 2000 backward-forward cycles were not sufficient to reach the goal SBC.

\section{Acknowledgements}

The effect on resistance of the novel mutations in this study was presented in part at the 12th European AIDS Conference, 11-14 November 2009, Cologne, Germany and at the 8th European HIV Drug Resistance Workshop, 17-19 March 2010, Sorrento, Italy. GV has no conflict of interest in relation to this article. KVdB, EVC, PL, MVH, BVK, LB and HvV were employees of TibotecVirco during conduct of the study.

Funding: This research was supported by IAP research network grantnr. P6/ 03 of the Belgian government (Belgian Science Policy) and by SymBioSys, the Katholieke Universiteit Leuven, Center of Excellence on Computational Systems Biology, (EF/05/007)

\section{Author details}

${ }^{1}$ Tibotec-Virco, Beerse, Belgium. ${ }^{2}$-Biostat, Katholieke Universiteit Leuven and Universiteit Hasselt, Belgium. ${ }^{3}$ VircoLab Inc., Chapel Hill, NC, USA.

\section{Authors' contributions}

$\mathrm{KVdB}$ designed the linear regression modeling approach, performed statistical analyses to derive a list of novel resistance-associated mutations, and drafted the manuscript. EVC and PL conceived of the study and assisted in its design. MVH assisted in interpreting the results and critically revised the manuscript. BVK coordinated the Virco lab work of the site-directed mutants and interacted with Medigenomix. LB participated in study design. GV advised on statistical analyses and helped draft the manuscript. HvV contributed to the design of the study and edited the manuscript. All authors read and approved the final manuscript.

Received: 11 April 2011 Accepted: 3 October 2011

Published: 3 October 2011

\section{References}

1. Vermeiren H, Van Craenenbroeck E, Alen P, Bacheler L, Picchio G, Lecocq P: Prediction of HIV-1 drug susceptibility phenotype from the viral genotype using linear regression modeling. J Virol Methods 2007, 145:47-55.

2. Rhee SY, Taylor J, Wadhera G, Ben-Hur A, Brutlag DL, Shafer RW: Genotypic predictors of human immunodeficiency virus type 1 drug resistance. Proc Natl Acad Sci USA 2006, 103(46):17355-17360.

3. Wang K, Jenwitheesuk E, Samudrala R, Mittler JE: Simple linear model provides highly accurate genotypic predictions of HIV-1 drug resistance. Antivir Ther 2004, 9(3):343-352.

4. Kutner MH, Neter J, Nachtsheim CJ, Li W: Applied Linear Statistical Models McGraw Hill; 2004

5. Hastie T: The Elements of Statistical Learning Springer; 2001.

6. Akaike H: A new look at the statistical model identification. IEEE Trans Automat Contr 1974, 19:716-723.

7. Schwarz G: Estimating the dimension of a model. Ann Stat 1978, 6(2):461-464.

8. Breiman L: Bagging Predictors. Machine Learning 1996, 24(2):123-140.

9. Efron B, Tibshirani R: An Introduction to the Bootstrap Chapman and Hall/ CRC; 1994 .

10. Austin P, Tu J: Bootstrap Methods for Developing Predictive Models. Am Stat 2004, 58:131-137.

11. Burman P: A Comparative Study of Ordinary Cross-Validation, v-Fold Cross-Validation and the Repeated Learning-Testing Methods. Biometrika 1989, 76(3):503-514.

12. Shao J: Linear Model Selection by Cross-Validation. J Am Stat Ass 1993, 88:486-494.

13. Zhang P: Model Selection Via Multifold Cross Validation. Ann Stat 1993, 21:299-313.

14. Tambuyzer L, Azijn H, Rimsky LT, Vingerhoets J, Lecocq P, Kraus G, Picchio G, de Béthune MP: Compilation and prevalence of mutations associated with resistance to non-nucleoside reverse transcriptase inhibitors. Antivir Ther 2009, 14:103-109.

15. Sevin AD, DeGruttola V, Nijhuis M, Schapiro JM, Foulkes AS, Para MF, Boucher CA: Methods for Investigation of the Relationship between Drug-Susceptibility Phenotype and Human Immunodeficiency Virus Type 1 Genotype with Applications to AIDS Clinical Trials Group 333. J Infect Dis 2000, 182:59-67.

16. van Rijsbergen C: Information Retrieval Butterworths, London; 1979.

17. Vingerhoets J, Peeters M, Azijn $H$, Tambuyzer L, Hoogstoel A, Nijs S, de Béthune MP, Picchio G: An update of the list of NNRTI mutations associated with decreased virologic response to etravirine: multivariate analyses on the pooled DUET-1 and DUET-2 clinical trial data. Antivir Ther 2008, 13(Suppl 3):A26.

18. Verlinden $Y$, Vermeiren $H$, Lecoca $P$, Bacheler L, McKenna $P$, Vanpachtenbeke M, Horvat LI, Van Houtte M, Stuyver LJ: Assessment of the antivirogram ${ }^{\circledast}$ performance over time including a revised definition of biological test cut-off values. Antivir Ther 2005, 10:S51.

19. Van Houtte M, Van Walle I, Van der Borght K, Muyldermans G, Pattery T, Bacheler L, Sista P: Rare HIV-1 drug resistance mutations exert subtle synergistic and antagonistic effects in the context of the genetic background. Rev Antivir Ther Infect Dis 2010, 1:2. 
20. Nikolenko GN, Delviks-Frankenberry KA, Palmer S, Maldarelli F, Fivash MJ, Coffin JM, Pathak VK: Mutations in the connection domain of HIV-1 reverse transcriptase increase 3 '-azido-3'-deoxythymidine resistance. Proc Natl Acad Sci USA 2007, 104:317-322.

21. Paredes R, Bannister W, Cozzi-Lepri A, Pou C, Bellido R, Bogner J, Gargalianos P, Banhegyi D, Clotet B, Lundgren JD, the EuroSIDA Study Group: Mutation A376S in the RT Connection Domain Is Associated with an Increased Risk of Virological Failure to Nevirapine-Based Therapy in NNRTI-Naïve HIV-Infected Subjects in the EuroSIDA Study. 16th Conference on Retroviruses and Opportunistic Infections, Montreal, Canada 2009, Abstract 646

22. Steegen K, Bronze M, Van Craenenbroeck E, Winters B, Van der Borght $K$, Wallis $C L$, Stevens W, Rinke de Wit TF, Stuyver $L$, the ART-A consortium: A comparative analysis of HIV drug resistance interpretation based on short reverse transcriptase sequences versus full sequences. AIDS Res Ther 2010, 7:38.

23. Whitcomb JM, Huang W, Limoli K, Paxinos E, Wrin T, Skowron G, Deeks SG, Bates M, Hellmann NS, Petropoulos CJ: Hypersusceptibility to nonnucleoside reverse transcriptase inhibitors in HIV-1: clinical, phenotypic and genotypic correlates. AIDS 2002, 16(15):F41-F47.

24. Clark SA, Shulman NS, Bosch RJ, Mellors JW: Reverse transcriptase mutations $118 \mathrm{I}, 208 \mathrm{Y}$, and $215 \mathrm{Y}$ cause HIV-1 hypersusceptibility to nonnucleoside reverse transcriptase inhibitors. AIDS 2006, 20(7):981-984.

25. Picchio G, Vingerhoets J, Parkin N, Azijn H, de Bethune MP: Nucleosideassociated mutations cause hypersusceptibility to etravirine. Antivir Ther 2008, 13(Suppl 3):A25.

26. Byrnes WW, Emini EA, Schleif WA, Condra JH, Schneider CL, Long WJ, Wolfgang JA, Graham DJ, Gotlib L, Schlabach AJ: Susceptibilities of human immunodeficiency virus type 1 enzyme and viral variants expressing multiple resistance-engendering amino acid substitutions to reserve transcriptase inhibitors. Antimicrob Agents Chemother 1994, 38(6):1404-1407.

27. Larder BA: $3^{\prime}$-Azido-3'-deoxythymidine resistance suppressed by a mutation conferring human immunodeficiency virus type 1 resistance to nonnucleoside reverse transcriptase inhibitors. Antimicrob Agents Chemother 1992, 36(12):2664-2669.

28. Vingerhoets J, Azijn H, Fransen E, De Baere I, Smeulders L, Jochmans D, Andries $K$, Pauwels $R$, de Béthune MP: TMC125 displays a high genetic barrier to the development of resistance: evidence from in vitro selection experiments. J Virol 2005, 79(20):12773-12782.

29. Cohen RA: Introducing the GLMSELECT PROCEDURE for Model Selection. SUGl 31 Proceedings, San Francisco, CA 2006, Paper 207-31.

30. Zhang P: On the Distributional Properties of Model Selection Criteria. J Am Stat Ass 1992, 87:732-737.

31. Hertogs $K$, de Béthune MP, Miller V, Ivens $T$, Schel $P$, Van Cauwenberge $A$, Van Den Eynde C, Van Gerwen V, Azijn H, Van Houtte M, Peeters F, Staszewski S, Conant M, Bloor S, Kemp S, Larder B, Pauwels R: A rapid method for simultaneous detection of phenotypic resistance to inhibitors of protease and reverse transcriptase in recombinant human immunodeficiency virus type 1 isolates from patients treated with antiretroviral drugs. Antimicrob Agents Chemother 1998, 42(2):269-276.

32. Perno CF, Svicher V, Ceccherini-Silberstein F: Novel drug resistance mutations in HIV: recognition and clinical relevance. AIDS Rev 2006, 8(4):179-190.

33. Shafer RW, Schapiro JM: HIV-1 drug resistance mutations: an updated framework for the second decade of HAART. AIDS Rev 2008, 10(2):67-84.

34. Winters B, Villacian J, Van Craenenbroeck E, Bacheler L: Development of vircoTYPE HIV-1 resistance analysis, including clinical cutoffs for TMC125, a new NNRTI. 15th Conference on Retroviruses and Opportunistic Infections, Boston, MA 2008, Abstract 873.

35. Winters B, Van Craenenbroeck E, Van der Borght K, Lecocq P, Villacian J, Bacheler L: Clinical cut-offs for HIV-1 phenotypic resistance estimates: update based on recent pivotal clinical trial data and a revised approach to viral mixtures. J Virol Methods 2009, 162(1-2):101-108.

doi:10.1186/1471-2105-12-386

Cite this article as: Van der Borght et al:: Cross-validated stepwise regression for identification of novel non-nucleoside reverse transcriptase inhibitor resistance associated mutations. BMC Bioinformatics 2011 12:386.

\section{Submit your next manuscript to BioMed Central and take full advantage of:}

- Convenient online submission

- Thorough peer review

- No space constraints or color figure charges

- Immediate publication on acceptance

- Inclusion in PubMed, CAS, Scopus and Google Scholar

- Research which is freely available for redistribution

Submit your manuscript at www.biomedcentral.com/submit
C Biomed Central 